\title{
Vécu des avortements à Ouagadougou : causes et conséquences socio- psychologiques.
}

\author{
Aline KAGAMBEGA \\ Département de Sociologie \\ Université Ouaga $1 \mathrm{Pr}$. Joseph KI-ZERBO \\ kag aline@yahoo.fr
}

\section{Résumé}

L'avortement est répandu au Burkina Faso, particulièrement dans la ville de Ouagadougou. Pour connaître les causes et conséquences socio-psychologiques des avortements, nous avons réalisé une étude dans la dite ville. L'objectif de cette étude était de comprendre les causes des avortements et leurs conséquences socio-psychologiques sur les femmes puis proposer des ajustements sur les approches déjà opérationnelles. Au total, 32 entretiens individuels et une observation non participante ont été réalisés auprès des femmes qui ont eu un avortement dans le passé, leurs proches et des professionnels de la santé. Les résultats ont montré que les déterminants des avortements sont les relations illégitimes, les raisons d'ordre économiques, les grossesses trop rapprochées, le refus de paternité, le non-respect des clauses définies au début de la relation et les grossesses issues de relations occasionnelles sans aucun projet de mariage, ni d'enfant. Des perturbations liées aux modifications des relations entre les deux partenaires, entre les femmes ayant avorté et les membres de leur famille, puis des stigmatisations, violences et sanctions de la part des agents de santé ont été relevées. Plusieurs symptômes dépressifs ont été rapportés (regret, honte, douleurs morales, insomnies, crainte, incertitude par rapport à une future grossesse, sentiment de désespoir). Des sentiments de satisfaction ont été aussi relevés. Les effets néfastes sociaux et psychologiques des avortements sont considérables et nécessitent une prise en charge des femmes en tenant compte de leurs besoins. II est de ce fait nécessaire de prévoir une prise en charge incluant les aspects médicaux, sociaux et psychologiques.

Mots clés: Causes des avortements, Conséquences socio-psychologiques, Ouagadougou.

\section{Abstract}

Abortion is widespread in Burkina Faso, especially in the city of Ouagadougou. To assess the causes of abortions and their socio-psychological consequences, we have conducted a study in this City. This paper aims to understand causes of abortions and their socio-psychological consequences on women who have experienced it and then propose corrective measures for the already existing operational approaches. On the whole 32 individual interviews and one non-participating observation were conducted with women who have already had an abortion, their relatives and healthcare professionals. Results showed that abortions causes include forced sexual intercourses, economic constraints, a series of pregnancies at short intervals, the non-respect of initial clauses of their relations, pregnancies from occasional intercourses without any project of marriage or child. Conflicts due to the change in the relationship between both partners, between aborted women and their families, stigmatizations, violence and sanctions imposed by health practitioners were also recorded. Several depressive symptoms were reported (regret, shame, moral pains, insomnia, fears, uncertainty as to future pregnancy, despair). Satisfaction feelings have also been mentioned. Social and psychological consequences of abortions are significant and require an appropriate care of women taking their needs into account. It is therefore necessary to develop a care system that includes medical, social and psychological aspects.

Keywords: Abortion causes, Socio-psychological consequences, Ouagadougou.

\section{Introduction}

Selon l'Organisation Mondiale de la Santé (OMS), on estime à 46 millions le nombre de grossesses interrompues volontairement chaque année dans le monde, dont 19 millions dans des conditions estimées dangereuses. Dans le monde, environ I3\% des décès maternels sont liés à des complications d'un avortement provoqué pratiqué dans de mauvaises conditions. $\mathrm{Ce}$ qui représente probablement près de 68000 décès par an (OMS, 2004 : 10). L'amélioration de la santé est l'une des 
préoccupations principales des autorités sanitaires dans le monde et plus particulièrement en Afrique. Dans les pays en développement, le risque de décès par suite des complications liées à un avortement pratiqué dans de mauvaises conditions est largement supérieur au risque lié à un avortement pratiqué par des professionnels dans de bonnes conditions (OMS, 2004 : 10). L'Afrique n'est pas en marge de cette situation. Une étude récente réalisée en Afrique de l'Est au Kenya en 2012 a estimé que 266 femmes kenyanes meurent pour 100.000 avortements non sécurisés (African Population and Health Research Center, Ministry of Health, Kenya, Ipas, and Guttmacher Institute, 2013:7). L'Afrique de l'Ouest est directement touchée. Au Togo, N'Bouke (20II) a montré que le recours à l'avortement est à la hausse à Lomé. Bien que l'estimation de son ampleur dépende de la méthode utilisée, l'auteur observe que plus de $32 \%$ des femmes ayant déjà été enceintes ont déclaré avoir avorté au moins une fois. Selon Bankole et al. (2013 : I0), au Burkina Faso, en 20I2, il y avait environ 105000 avortements. Le taux d'avortement qui avait été estimé à Ouagadougou, était de 28 pour I 000 femmes de 15 à 49 ans. II était plus élevé (42 pour I 000) à Bobo- Dioulasso. Le taux était inférieur (22 pour I 000$)$ dans les zones rurales, où vit $75 \%$ de la population. Sedgh et al. (201I: 147) avaient estimé le nombre d'avortements à 87200 par an en 2008. Cette estimation est passée à 105000 en 20/2. La proportion des avortements à risque par rapport au total des avortements serait en hausse, passant de $44 \%$ en 1995 à $47 \%$ en 2003 puis à $49 \%$ en 2008 (Zongo et al., 2015 : 12). Le cumul des données sur les avortements des rapports du Ministère de la santé de 2008 à 2015 compilés par la Direction Générale de l'Information et des Statistiques Sanitaires (DGISS) révèle qu'en moins d'une décennie, il $y$ a eu officiellement 206525 avortements pour l'ensemble du pays, dont 31 I42 dans la Région du Centre, cheflieu Ouagadougou.

Ces données épidémiologiques cachent des situations individuelles avec d'importantes conséquences sociales et psychologiques notamment pour les femmes. En rappel, la question de l'avortement, fait l'objet d'une forte réprobation sociale et est illégale. Une enquête sur le jugement moral envers certains phénomènes sociaux au Burkina Faso du Centre pour la Gouvernance Démocratique (CGD) a mesuré les comportements qui sont jugés inadmissibles en société. Selon l'enquête de 2007, parmi ces comportements figurent en première position l'homosexualité (87\%), ensuite la prostitution (86\%) et en troisième position l'avortement (85\%). La même étude menée trois ans après a donné des résultats similaires : les comportements répréhensibles restent dans l'ordre l'homosexualité (88\%), la prostitution et l'avortement viennent en deuxième position (avec chacun) (86\%) (CGD, 2010 : 26). De plus, l'avortement est interdit par les religions de la majorité des Burkinabè dont $60,05 \%$ se déclarent musulmans, $23,2 \%$ chrétiens $(19,0 \%$ de catholiques ; $4,2 \%$ de protestants) et $15,3 \%$ animistes (INSD, 2009: 93). De ce fait, les injonctions religieuses contenuent dans les Livres Saints dont se réfère la majorité de la population - le Coran, la Bible - qui condamnent l'avortement ont un fort écho dans les valeurs populaires. Dans la Bible, il est mentionné dans Jérémie chapitre I, verset 5, qu' " Avant de te former dans le ventre de ta mère, je te connaissais, et avant que tu naisses, je t'avais consacré...» ; ceci pour signifier l'omniprésence de Dieu dans toutes les étapes de l'évolution de l'être humain (Société biblique de Genève, 2007). Par ailleurs, l'interprétation d'un certain nombre de sourates du Coran permet de comprendre que l'avortement provoqué est condamné (communication personnelle - Imam d'une mosquée de Ouagadougou - le 12 Novembre 2013). Ces interdictions religieuses sont renforcées par celles contenues dans le Code Pénal selon lesquelles l'avortement est interdit par la loi, exception faite pour les causes thérapeutiques, de viol et d'inceste (voir les articles 383 à 386 et 388 à 390 du code pénal). En outre, la loi $N^{\circ} 049-2005 / A N$ portant sur la santé de la reproduction du 2I décembre 2005 dispose en son article 20 que : «Toutes les techniques et méthodes de planification familiale, à l'exception de l'avortement provoqué ou interruption volontaire de grossesse, sont autorisées dans les formations sanitaires publiques et privées qui remplissent les conditions requises ". Cette condamnation de la pratique de l'avortement et les nombreux problèmes liés à l'utilisation des contraceptifs créent un cercle vicieux de grossesses non désirées et d'avortements provoqués à répétition conduisant parfois à des décès. Le drame est que les avortements provoqués constituent l'un des facteurs de morbidité et de mortalité maternelles. Selon le Ministère de la santé (201 I: 30 ), au Burkina Faso, 10\% des décès maternels étaient dus aux avortements. Aussi, en 2014, 50 femmes sont décédées suite à des complications d'un avortement au Burkina Faso, dont 25 dans la région du Centre, région dont fait partie Ouagadougou (Direction générale des études et des statistiques sectorielles du ministère de la santé, 2015 : 145). De même, des études hospitalières ont montré que les avortements étaient responsables d'un grand nombre de décès maternels. Dao (2010:48) a montré dans son étude menée sur onze ans ( $I^{\text {er }}$ Octobre 1997 au 3I Décembre 2007) dans le service de gynécologie 
obstétrique du Centre Hospitalier Universitaire Yalgado Ouédraogo (CHU-YO) que sur 129 cas de décès enregistrés, 93 cas de décès $(72,09 \%)$ étaient liés à l'avortement provoqué. Les facteurs explicatifs de ces forts taux de décès sont entre autres le non recours ou le recours tardif aux soins de santé. Rossier et al. (2006 : 254) qui ont évalué la prévalence des avortements par l'invention de la méthode nommée "méthode des confidentes" avaient relevé que $60 \%$ des femmes qui avaient eu un avortement provoqué avaient été atteintes dans leur santé et $14 \%$ seulement parmi celles-ci étaient soignées dans un centre de santé de référence de la ville.

Conscient des difficultés que rencontrent les femmes à la suite d'un avortement et du taux de décès lié à ce phénomène, l'Etat burkinabè a pris des mesures pour réduire les complications avec notamment l'introduction en 1997 du concept de Soins Après Avortement (SAA). Cependant, cette politique de l'Etat ne prend en charge que de façon partielle les avortements. En effet, la prise en charge est essentiellement médicale. Les aspects psychosociaux étant négligés. Concrètement, il n'existe aucune structure de prise en charge psycho-sociale des femmes ayant avorté. Ce qui n'est pas sans avoir des conséquences sur la vie de ces femmes. Une pré enquête que nous avions menée avait révélée que beaucoup de femmes qui avortent à Ouagadougou tombent dans la détresse et ont du mal à se ressaisir.

Ainsi, l'ampleur des avortements, le problème social que cela cause et l'insuffisance d'étude menée au plan national spécifiquement sur le vécu de l'avortement par les femmes elles-mêmes, les interactions des femmes qui ont avorté avec les institutions sanitaires de prise en charge et leur entourage sont autant de facteurs qui ont motivé une étude sur ce domaine.

La question générale de recherche est la suivante :

Quels sont les déterminants des avortements et leurs conséquences socio-psychologiques sur les femmes?

L'objectif visé est de comprendre les causes des avortements et leurs conséquences sociopsychologiques sur les femmes puis proposer des ajustements afin de contribuer à améliorer les approches déjà opérationnelles.

Autour de cet objectif général se greffent trois objectifs spécifiques:

I. Identifier les causes des avortements.

2. Analyser les conséquences sociales des avortements sur les femmes.

3. Analyser les récits de vie permettant de décrire les ressentis des femmes envers elles-mêmes après l'acte d'avortement provoqué.
Après la partie introductive, nous traiterons de la revue de littérature, du cadre théorique et conceptuel, puis il sera question de la méthodologie, des résultats et discussion avant la conclusion.

\section{REVUE DE LITERRATURE}

Les causes des avortements et leurs conséquences sanitaires

Une revue systématique de la littérature a relevé que les causes des avortements sont entre autres le désir de terminer ses études, le déni de la paternité, le sentiment d'être très jeune pour avoir un enfant, la pauvreté, la peur de la réaction des parents, la crainte de l'entourage, la convenance personnelle, les grossesses illégitimes, le statut social, les avortements réalisés pour des problèmes de santé. L'avortement est aussi utilisé comme une méthode contraceptive dans le but de réguler les naissances.

Une étude, déjà ancienne, menée à Génève par Kellerhals (1977 : 333 et 334) a soutenu que les motifs du recours à l'avortement s'établissaient ainsi : $14 \%$ pour atteinte jugée grave à la santé physique et psychique de la mère, $30 \%$ environ pour détresse socio-économique profonde, et une majorité de cas où seul des "micro-équilibres" personnels et relationnels étaient en cause (sentiment de solitude ou d'immaturité, aspirations professionnelles déterminantes, relation conjugale vacillante, volonté de se définir autrement que par l'enfant, etc.).

Dans le contexte ouest-africain, pour Guillaume (2003) dans son étude menée à Abidjan en Côte d'Ivoire, les deux principaux motifs avancés pour recourir à l'avortement sont d'abord la poursuite des études, et, ensuite, la crainte de la réaction des parents mentionnée par les jeunes femmes. Ces jeunes femmes "cachent " à leur entourage une sexualité hors union socialement stigmatisée, que cette grossesse dévoilerait. C'est donc la situation matrimoniale, c'est-à-dire le fait de ne pas être mariée ou d'être dans une union peu stable, ainsi que le refus du père de reconnaître l'enfant qui expliquent le recours à l'avortement. Toutefois, les raisons économiques (coût d'un enfant ou impossibilité de poursuivre la grossesse en raison de l'activité de la mère) ainsi que la volonté de limiter ou d'espacer les naissances sont également citées comme motifs d'avortement, mais plutôt de la part des femmes en union stable. En effet, certaines femmes ont expliqué leur avortement par un besoin de limiter (3\%) ou d'espacer $(9 \%)$ les naissances, exprimé par le jugement que "le dernier enfant était trop petit". Pour ces femmes, avortement et contraception sont deux outils de régulation de la fécondité utilisés de façon complémentaire. L'avortement fait suite à l'échec d'une méthode contraceptive, souvent une méthode naturelle, ou c'est l'expérience d'un 
avortement qui les incite à utiliser la contraception. L'enquête a révélé que $(30 \%)$ des femmes avaient utilisé à la fois la contraception et l'avortement pour éviter toute grossesse non prévue ou non désirée.

Toujours en Côte d'lvoire, d'autres auteurs, Benie Bi et al. (2012:67 et 70) ont également trouvé une liaison entre l'utilisation des contraceptifs et le recours à l'avortement. Étant donné que l'utilisation d'une contraception traduit le désir des femmes de ne pas débuter de grossesse, leur attitude serait de recourir fréquemment à l'avortement en cas de grossesses non désirées. Les motifs les plus évoqués par les personnes pour justifier le recours à l'avortement étaient dominés par la crainte de la réaction des parents $(27,7 \%)$, l'âge trop jeune pour avoir un enfant $(22,2 \%)$, le manque de ressources financières pour assurer la charge d'un enfant $(2 \mid, 3$ $\%)$ et la volonté de poursuivre les études (15,5\%).

En outre, une étude menée à Lomé au Togo par N'Bouke et al. (2012:375 et 376) a montré que l'avortement est pratiqué pour réguler la fécondité. En effet, bien que l'avortement ne soit pas le mode de réduction de la fécondité le plus important, leurs résultats montrent qu'au moins 0,34 naissance par femme a été évitée par le recours à l'avortement en 2002 , soit une réduction d'au moins $10,8 \%$ de la fécondité potentielle des femmes de 15 à 44 ans. Pour l'auteur, ces résultats confirment l'idée selon laquelle, bien que la législation sur l'avortement soit restrictive au Togo et qu'il $y$ ait une condamnation sociale de l'acte, une partie de la régulation des naissances est assurée par des avortements.

L'étude conduite à Rabat au Maroc par Bakass et Ferrand $(2013: 60)$ ne déroge pas à la règle. Ces auteurs ont soutenu que la stigmatisation des naissances hors mariage est si forte que pour la quasitotalité des personnes interrogées, quels que soient leur sexe et leurs appartenances religieuses, le recours à l'avortement en cas de grossesse indésirable paraît toujours envisageable, voire souhaitable.

Au Burkina Faso, Guiella (2004 : 12 et 13) a trouvé dans son étude, des déterminants similaires. En effet, il estime que les raisons les plus fréquemment évoquées pour expliquer le recours aux avortements clandestins sont de deux ordres. La première cause est économique et la seconde est d'ordre social. Dans une société où la sexualité reste un tabou, les jeunes rencontrent l'hostilité de la société et de la famille qui acceptent difficilement qu'une fille non mariée soit enceinte. Ces situations sont perçues dans la société comme un déshonneur de la famille et cela peut parfois entraîner le bannissement de la fille "fautive » de cette famille.

Ouédraogo (2015) partage cette opinion. Cette auteure soutient que c'est la peur de perdre un soutien dans un contexte de dépendance et la crainte des implications du statut de "fille-mère » qui conduisent à opter pour l'avortement. En effet, le statut de "fille-mère ", une notion qui revêt une connotation péjorative, expose à la stigmatisation et au rejet, et surtout, il réduit les chances de trouver un mari car la maternité détruit la «fraicheur » recherchée par les hommes. Le recours à l'avortement devient alors un moyen pour préserver les liens sociaux et économiques. Dans d'autres cas, la femme fléchit parce que l'homme renie tout simplement la responsabilité de la grossesse, ou disparaît à son annonce. Elle renonce à poursuivre la grossesse dans ces conditions, l'avortement devenant la seule alternative pour échapper à la précarité économique et sociale, et pour être en phase avec les normes procréatives (pas d'enfant sans père par exemple) afin d'optimiser ses chances de construire une nouvelle relation.

Les principales complications varient selon les études.

En Côte d'Ivoire, Benie Bi et al. (2012 : 67 et 72) dans leur travail de recherche menée sur l'épidémiologie des avortements provoqués en Côte d'Ivoire ont montré que plus de la moitié $(55,8 \%)$ des femmes avaient déclaré avoir déjà eu des complications qui sont plus fréquentes à l'issue des avortements à domicile que ceux effectués en milieu hospitalier. La fréquence des complications post abortum était de 55,2\%. Ces complications étaient dominées par les douleurs pelviennes chroniques $(68,2 \%)$, et plus dramatique, les perforations (58 $\%)$, les infections $(17,6 \%)$ et les hémorragies (16 $\%)$.

Au Togo, N'Bouke (201I: 134) dans sa thèse traitant du recours à l'avortement provoqué à Lomé fait remarquer que les avortements souvent pratiqués par des personnes non qualifiées ne sont pas sans conséquences sur la santé des femmes. Plus de $60 \%$ des femmes avaient déclaré avoir eu des complications sanitaires après leur plus récent avortement. Avec la possibilité d'avoir en même temps plusieurs conséquences physiques, les complications les plus fréquemment observées auprès des femmes qui en ont eu, étaient des hémorragies (72\%), des maux de reins (16\%), des infections $(6,5 \%)$ et des maux de bas ventre $(5,7 \%)$. II apparaît que $88 \%$ des femmes s'étaient fait hospitaliser dans le cadre du traitement des complications d'avortement. La prise en charge consistait souvent à l'administration des soins appropriés et spécifiques aux complications, à des transfusions sanguines ou à des opérations chirurgicales. Presque la totalité $(94,4 \%)$ des femmes qui ont été hospitalisées suite à une complication de 
leur avortement avait subi une opération dans le cadre du traitement de la complication.

Au Burkina Faso, des études qui se sont penchées sur cette thématique ont relevé des résultats similaires. En rappel, Rossier et al. (2006: 254) avaient révélé que $60 \%$ des femmes qui avaient eu un avortement provoqué avaient été atteintes dans leur santé et seulement $14 \%$ de ces femmes étaient soignées dans un centre de santé de référence de la ville. Le faible effectif des femmes qui ont eu recours aux centres de santé de référence dans l'étude de Rossier et al. (2006 : 254) pour leur prise en charge post-avortement pourrait s'expliquer non seulement par la crainte de réprésailles compte tenu du fait que l'avortement provoqué clandestin est réprimé par la loi au Burkina Faso, mais aussi par le fait que les populations burkinabè ont une attitude néfaste vis-àvis de l'avortement provoqué.

Si dans cette étude, très peu de femmes (I4\%) qui avaient été atteintes dans leur santé étaient soignées dans un centre de santé de référence de la ville, N'Bouke (20II: 135) avait soutenu que les complications d'avortement semblaient avoir été prises en charge dans des centres de santé. En effet, $94 \%$ des femmes qui ont eu des complications à l'issue de leur avortement s'étaient rendues dans des hôpitaux pour se faire soigner et 1,9\% s'étaient rendues dans des cliniques privées.

Une autre étude qui s'est intéressée aux causes et aux conséquences de la grossesse non désirée et de l'avortement provoqué au Burkina Faso a souligné que les professionnels de santé selon leur estimation ont déclaré que quatre femmes qui avortent sur 10 $(4,3 \%)$ doivent faire face à des complications. Une estimation fondée sur les réponses des femmes ellesmêmes suggère que ce chiffre est de $49 \%$. La proportion des avortements engendrant des complications est considérée comme plus élevé chez les femmes qui ont recours à un praticien traditionnel (57\%). La deuxième stratégie la plus dangereuse est la pratique de l'avortement par la femme elle-même. Il a été estimé que la moitié de ces femmes (5l\%) connaissent des complications. Même parmi les femmes qui ont recours à des personnels de santé qui ne sont pas des médecins, les auteurs pensent qu'une sur cinq subit des complications (2I-23\%) (Bankolé et al. 2013 : 20).

Dans l'étude de Ouédraogo (2014: 63), les principales complications étaient les hémorragies $(51,2 \%)$, les infections $(37,4 \%)$, l'insuffisance rénale $(14,4 \%)$. Dans celle de Palenfo (20I5: 69 et 70 ), les complications étaient dominées par l'anémie $(83,80 \%)$, l'infection $(67,51 \%)$, l'hépatonéphrite (lésions touchant le tissu rénal) $(37,8 \%)$, les troubles de la conscience $(10,8 \mid \%)$ et l'hématémèse (le fait de vomir du sang) $(8,1 \%)$.
Dans la série de Ouandaogo (2015), l'anémie $(75,19 \%)$ était la complication la plus dominante, suivi de l'endométrite $(18,6 \%)$, les états de choc $(4,4 \%)$ et l'insuffisance rénale $(I, 93 \%)$.

Ces études antérieures n'ont pas suffisamment mis l'accent sur les conséquences sociopsychologiques des avortements provoqués à Ouagadougou, d'où l'intérêt de contribution qui intègre en particulier ces aspects. II urge alors d'analyser ces conséquences dans ladite ville afin de tirer des leçons et contribuer à réorienter au mieux la politique de prise en charge des avortements mise en œuvre par l'Etat Burkinabè.

\section{CADRE THEORIQUE ET CONCEPTUEL}

Le cadrage théorique adopté dans cette investigation se rattache au courant théorique de Goffman qui étudie le comportement humain. Goffman pense en termes de "relations syntaxiques qui unissent les actions de (s) diverses personnes mutuellement en présence "(Winkin, 1999: 94 et 96). En effet, quand des acteurs sont en situation de coprésence physique, ils sont soumis à un ensemble de règles interactionnelles qui intègrent dans le cadre de notre sujet les relations qui se nouent entre les femmes qui ont avorté et d'autres acteurs qui sont entre autres leur mari ou leur ami, leur famille, leur proche, les prestataires de soins après avortement. Nous focalisons notre analyse sur les actions réciproques entre ces acteurs sus-cités.

Dans le cadre de cette étude, l'avortement est la mort de l'embryon ou du fœetus au cours de son développement. L'avortement provoqué est le fait de mettre volontairement fin à la vie de l'embryon ou du fœetus. Il est le contraire de l'avortement spontané ou fausse couche.

L'avortement provoqué peut être thérapeutique (pour soustraire la mère à un danger que la grossesse est sensé lui faire courir), eugénique (pour raison de présence d'une tare génétique, héréditaire ou congénitale ou suspecte de l'être chez le fotus), social (en cas de raison sociale grave : viol, inceste) quand il est autorisé par la loi. II s'oppose à l'avortement provoqué clandestin (APC).

Les conséquences sociales sont les interactions/relations entre la femme ayant avorté et son conjoint/partenaire ; entre la femme et sa famille ; entre la femme et son entourage ; entre la femme et son agent avorteur après l'avortement.

Les conséquences psychologiques sont les faits existants à un instant donné et déterminant la conduite d'une personne ou d'un groupe à ce moment précis.

Des expressions en langue moore 
«pœs yiisg $s^{\underline{a}}$ n $t \circledR$ ne laloa », expression en moore, littéralement traduite en langue française par « les avortements autorisés par la loi ».

«pœs@ $s^{\underline{a}}-$ solgd $n$ yiisd ${ }^{\underline{a}} w \odot$ », expression en moore, littéralement traduite en langue française par " les grossesses qu'on se cache pour avorter-là ».

Ces expressions sont traduites par un concepteur de la Direction de la recherche des innovations en éducation non formelle et en alphabétisation (DRINA), ex Institut National d'Alphabétisation (INA).

\section{METHODOLOGIE}

\section{Ouagadougou : caractéristique générale}

Ouagadougou est la capitale politique du Burkina Faso située dans la province de Kadiogo et dans la région du Centre. Suite au redécoupage de la Commune de Ouagadougou en 12 Arrondissements et 55 secteurs consacré par la « loi 066-2099/AN du 22 décembre 2012 portant découpage des Communes urbaines à statut particulier au Burkina Faso ", il ressort que Ouagadougou compte I.915.102 habitants avec $49.60 \%$ de femmes et $50,40 \%$ d'hommes (Direction des Etudes et de la Planification (DEP), $2012: 6$ ).

Nous avons choisi de conduire l'enquête dans la ville de Ouagadougou parce que cette ville regorge beaucoup plus de formations sanitaires comparativement aux autres villes du pays. Cette ville dispose d'une assez bonne couverture sanitaire qui a permis de rencontrer et de discuter avec plusieurs catégories d'agents de santé de différentes formations sanitaires sur le sujet.

Le choix de mener l'enquête dans toute la ville de Ouagadougou a été guidé par le souci d'augmenter notre chance de pouvoir entrer en contact avec la population cible car, étant donné que l'avortement est un domaine sensible, si nous délimitons un quartier de la ville, nous risquons de n'avoir pas assez de femmes qui ont avorté et qui acceptent de participer à l'enquête.

La collecte des données a été faite en deux (2) étapes :

La première étape a consisté à récolter des données secondaires à travers une revue de littérature relative aux avortements et des données primaires auprès des personnes et structures ressources. La recherche documentaire a concerné la recherche de travaux et d'auteurs pouvant permettre l'approfondissement de la réflexion sur les causes, conséquences sociales et psychologiques des avortements.

La seconde phase a consisté à la collecte des données primaires essentiellement par des entretiens individuels approfondis et une observation non participante auprès des femmes qui ont eu un avortement dans le passé, leurs proches, des professionnels de la santé, des personnes ressources, etc.

Une grille d'observation non participante et un guide d'entretien thématique ont été conçus et adaptés pour les différentes catégories d'informateurs.

Conformément aux objectifs de cette phase de l'évaluation, l'entretien individuel approfondi a été utilisé comme technique principale pour recueillir l'information, selon une démarche socioanthropologique. Une observation non participante a consisté en des visites préparatoires, à aller discuter plusieurs fois avec les personnes concernées par l'étude, notamment les femmes qui ont avorté, afin de nous familiariser à elles et d'avoir un statut reconnu. Ces visites ont permis de collecter des informations préliminaires par des prises de notes sur la base de discussions informelles avant les entretiens formels.

L'enquête a été purement qualitative. Le séjour prolongé sur le terrain a été très important pour permettre le croisement d'un maximum d'information et surtout parvenir à la saturation. La rigueur méthodologique n'a pas dépendu du nombre de personnes enquêtées, mais de l'enregistrement du maximum d'informations pertinentes et diversifiées. C'est ce que fait remarquer Olivier de Sardan (2005: 43) lorsqu'il souligne : "Chaque type d'enquête a ses formes de rigueur, c'est-à-dire ses formes spécifiques de validation ou de plausibilisation des données produites. Mais la rigueur de l'enquête de terrain n'est pas chiffrable, à la différence de la rigueur de l'enquête par questionnaire qui l'est en partie». De ce fait, pour l'échantillonnage, nous avons mis l'accent sur la diversité des enquêtés dans l'intention d'obtenir les avis des différentes catégories de personnes. Le nombre d'enquêtés a été déterminé à la fin de la recherche par le principe de la saturation.

Comme l'affirmaient Quivy et Van Campenhoudt (1995:184), l'entretien demeure l'outil le plus approprié pour recueillir ces types de données : " Ainsi, s'instaure en principe un véritable échange au cours duquel l'interlocuteur du chercheur exprime ses perceptions d'un évènement ou d'une situation, ses interprétations ou ses expériences tandis que par ses questions ouvertes, le chercheur facilite cette expression, évite qu'elle s'éloigne des objectifs de la recherche, et permet à son vis- à - vis d'accéder à un degré maximum d'authenticité et de profondeur».

Si le guide d'entretien a motivé notre choix, c'est qu'à la différence d'avec le questionnaire standardisé, le guide permet un processus itératif. Cela a permis 
de revoir l'approche du thème et de faire des feedback au cours de l'entretien.

Une enquête intensive socio-anthropologique a été faite en ayant recours à 32 enquêtés composés de sept femmes ayant fait des avortements clandestins, une femme ayant fait un avortement thérapeutique, deux avorteurs clandestins, quatre agents de santé, un agent de l'Association Burkinabè pour le Bien-être Familial (ABBEF), un chimiste, le partenaire d'une femme qui a avorté, deux témoins privilégiés (confidentes des femmes qui ont avorté), quatre personnes ressources (autorités religieuses et coutumière), un juge d'instruction, un agent du ministère de la santé publique, deux agents du Ministère de l'action sociale à la direction provinciale de l'action sociale et de la solidarité nationale du Kadiogo, un agent au niveau d'une représentation de l'action sociale détachée au sein de la Maison d'Arrêt et de Correction de Ouagadougou (MACO), quatre responsables d'association et d'Organisation Non Gouvernementale (ONG).

Les 32 entretiens individuels réalisés comprennent 3 I entretiens individuels approfondis et un entretien informel réalisé avec une fille qui a fait un avortement clandestin.

Les femmes qui ont avorté interrogées dans le cadre de cette étude sont majoritairement jeunes. La plus jeune avait 20 ans au moment de l'enquête et la plus âgée 40 ans.

La technique de la boule de neige a été utilisée pour produire les données, la technique de la triangulation pour croiser plusieurs sources d'information issues de tous les groupes stratégiques pouvant donner des informations concernant les avortements provoqués. Ces techniques ont été complétées par le principe de l'itération et de saturation d'information.

La technique de la boule de neige signifie que de chaque entretien est né de nouvelles pistes, de nouveaux interlocuteurs possibles, suggérés directement ou indirectement au cours de nos entretiens. Cette technique a permis de choisir l'ensemble des participants à l'enquête de façon raisonnée, en ciblant au préalable par des entretiens informels, les informateurs clés pouvant renseigner sur les centres d'intérêt explorés.

Ainsi, en ce qui concerne les femmes qui ont avorté, nous avons sollicité les entretiens avec elles suite à des informations que nous ont fournies leurs confidentes, leurs proches. Ces femmes ont été interrogées car elles sont les mieux placées pour parler de leur prise en charge et des relations sociales autour d'elles après l'avortement.

Quant aux confidentes des femmes interviewées, compte tenu du fait que les salons de coiffures sont généralement fréquentés par de jeunes femmes (femmes en âge de procréer), nous avons ciblé quelques coiffeuses avec lesquelles nous avons faits des entretiens approfondis parce que nous nous sommes dit que des femmes ayant avorté se confieraient à elles lors des causeries. Après la réalisation d'un entretien avec une coiffeuse, nous lui demandions de nous mettre en contact avec des femmes ayant avorté et qui allaient accepter participer à l'enquête.

Concernant les prestataires de soins, ils ont été choisis en fonction du rôle joué dans la formation sanitaire, notamment à la maternité où les femmes qui ont avorté sont prises en charge. Ces prestataires de soins après avortement ont été interviewés pour parler de leur vécu et de leurs opinions par rapport à la prise en charge des femmes, mais aussi de leurs rapports avec ces femmes ayant avorté.

Pour les personnes ressources, leur choix a été fait aussi de façon raisonnée, en ciblant par des entretiens informels les informateurs clés pouvant renseigner sur les centres d'intérêt explorés. Cela nous a permis de retenir des experts qui travaillent dans le domaine de la santé de la reproduction et des personnes qui ont une bonne connaissance des pratiques d'avortement dans un contexte socioculturel ou religieux. Leurs discours ont permis de comprendre la situation de certaines femmes qui ont avorté.

Les agents de la Direction de la Santé de la Mère et de l'Enfant (DSME) ont été ciblés en fonction de leur implication dans les activités de promotion de la santé de la reproduction. Ces agents, entant qu'acteurs de la prise en charge médicale entretiennent des relations concrètes avec les patientes. Ces relations sont fondées entre autre sur le respect des règles de l'éthique de leur profession.

Des autorisations délivrées par le Chef de Département de Sociologie de l'Université de Ouagadougou, par le Directeur régional de la Santé du Centre et le par Ministre de la justice, des droits humains et de la promotion civique, garde des sceaux ont permis de mener à bien l'étude.

Avec cette dernière autorisation, les agents de sécurité pénitentiaire se sont chargés de mettre les femmes incarcérées pour raison d'avortement à notre disposition.

Nous avons jugé utile de faire un entretien avec un juge d'instruction pour mieux comprendre les aspects juridiques, l'application de la loi dans le cadre des avortements.

Par ailleurs, nous avons interviewé un chimiste dans l'intention de savoir la composition de certains produits traditionnels (la poudre noire par exemple) utilisés par certaines de nos enquêtées pour provoqué l'avortement. Le type de produit utilisé pour provoquer l'avortement peut permettre 
d'analyser les conséquences socio-psychologiques dont souffrent certaines femmes qui ont avorté.

Nous avons de même interrogé des avorteurs clandestins pour qu'ils s'expriment au sujet des moyens utilisés pour avorter et de leurs perceptions de l'avortement. La rencontre avec ces acteurs dont on a rarement le témoignage a été possible grâce à leurs amis que nous avons côtoyés. Ceux-ci après avoir compris le bien fondé de l'étude ont accepté nous mettre en contact avec les avorteurs clandestins. Ces avorteurs à leur tour qui avaient été réticents au départ, avaient finalement accepté de nous accorder les entretiens car ils se sont dit que cette étude pouvait conduire à la légalisation de l'avortement qu'ils souhaitent un jour voir se réaliser au Burkina Faso. Les informations données par ces avorteurs nous ont permis d'analyser les conséquences socio-psychologiques dont souffrent certaines femmes ayant avorté.

Des agents du Ministère de l'action sociale ont été interviewés. En effet, la Direction provinciale de l'action sociale et de la solidarité nationale fait une prise en charge psychosociale informelle qui consiste essentiellement dans le cadre des avortements, à donner des conseils aux femmes qui ont avorté. Cela dans le but de les amener à se réssaisir. Les types de conseils varient en fonction du problème que chacune d'elle fait face. Par ailleurs, cette institution réfère si nécessaire, des femmes qui ont avorté pour une prise en charge dans des formations sanitaires avec lesquelles elle coopère. Ces agents nous ont parlé de leurs expériences concernant les femmes qui avortent.

Un agent de la représentation de l'action sociale située au sein de la MACO a été interviewé. Celui-ci nous a parlé du rôle joué par les agents de la dite institution installée au sein de la MACO dans le cadre des avortements.

Un agent de l'Association Burkinabè pour le Bien Etre Familial (ABBEF) a été interrogé parce que cette association est une société civile reconnue comme association d'utilité publique par le gouvernement du Burkina Faso et qui œuvre entre autres dans le domaine de la Santé de la Reproduction (SR). L'ABBEF dispose d'une clinique qui offre des soins post-avortement. L'agent interviewé nous a parlé des actions que l'association mène concernant les femmes ayant avorté.

D'autres associations et Organisations Non Gouvernementales (ONG) ont été concernées par l'étude. II s'agit de l'Associations Ampo MIA-ALMA, de l'Association d'Appui et d'Eveil Pugsada (A.D.E.P), de la Fondation Cardinal Emile Biayenga (FOCEB) et de l'Association CARMEN - KISITO qui œuvrent pour la réinsertion sociale des jeunes filles en difficulté à Ouagadougou. Les agents de ces associations et ONG nous ont parlé de leurs expériences concernant la prise en charge des cas d'avortements.

La technique de la triangulation signifie l'utilisation de plusieurs techniques, l'interrogation de plusieurs catégories de personnes, le croisement de plusieurs données provenant de différentes sources, la comparaison des données.

Cette triangulation des informations de personnes concernées par l'enquête (les femmes ayant avorté, les professionnels de la santé, les confidentes, etc.) ainsi que des sources d'information (revue documentaire, entretiens individuels approfondis, etc.) ont permis de confronter les différentes informations collectées afin d'éviter d'éventuel décalage.

La technique de l'itération a consisté à réécouter les entretiens ou à les retranscrire afin d'ajouter ou de reformuler certaines questions dans le souci d'être conforme aux objectifs spécifiques de l'étude.

La technique de la saturation indique que le chercheur arrête de recueillir des informations lorsqu'il n'apprend plus rien de nouveau.

L'enquête s'est s'achevée après la saturation des informations. Contrairement à l'enquête par questionnaire, cette méthode n'inclut pas dans son dispositif un signal de " fin ".

En recherchant l'avis de ces différentes catégories de personnes, nous recherchons un ensemble d'informations aussi complètes que possible, mais aussi une diversité de perspectives qui peuvent se renforcer, se compléter ou même se contredire.

Le traitement des données a été fait à l'aide de l'outil informatique. Concrètement, tous les entretiens formels intégralement enregistrés ont été transcrits à l'aide du logiciel de transcription numérique appelé "f4". Ces entretiens transcrits, ainsi qu'un entretien informel saisi ont été analysés à partir d'une classification thématique des extraits de discours.

Nous avons procédé ensuite à une analyse de contenu manuelle des discours des informateurs et une triangulation des données obtenues.

Une synthèse des différents thèmes dégagés en référence aux objectifs de cette phase d'enquête a été utilisée dans la présentation des résultats. En complément de cela, plusieurs extraits de discours significatifs ont été cités pour illustrer et interpréter.

\section{RESULTATS}

Les résultats mettent en exergue des données déjà plus ou moins connues, mais aussi des témoignages qui ne ressortent pas assez dans les études antérieures conduites à Ouagadougou, notamment les données portant sur les femmes incarcérées, les 
avorteurs, les conflits sournois entre avorteurs et avortés.

\section{Les déterminants ordinaires de l'avortement}

Notre étude relève quasiment les mêmes déterminants que ceux issus de travaux antérieurs évoqués plus haut.

L'avortement est reprouvé par les citoyens burkinabè de façon générale, cependant, la tendance est que c'est un phénomène qui est couramment pratiqué, malgré la sanction de la loi.

Dans notre étude, les causes des avortements provoqués sont notamment les relations illégitimes, les raisons économiques, les grossesses trop rapprochées, le refus de paternité, le non-respect des clauses définies au début de la relation, les grossesses issues des relations occasionnelles sans projet de mariage, ni d'enfant et les avortements pratiqués dans le but de sauver la vie de la mère.

\section{Le refus de paternité}

La majorité de nos enquêtées qui a avorté a évoqué le refus de paternité comme cause de l'avortement : "Moi je l'ai informé de ma situation et il a dit que la grossesse ne lui appartient pas. Comme il a dit que la grossesse ne lui appartient pas, je ne sais plus ce que je vais dire. Je vais dire que la grossesse appartient à qui ? Alors que moi je sais que c'est sa grossesse. II dit que ce n'est pas sa grossesse. Après cela, c'est sa maman même qui est allée voir ma copine et lui a dit de me dire d'avorter car la grossesse n'appartient pas à son fils ... A chaque fois que la vieille (la maman de l'auteur de la grossesse) me croisait, elle me causait sale alors que je ne peux rien lui dire. C'est ce qui m'a amené à avorter et à avoir la paix " (enquêtée non scolarisée ayant 20 ans, célibataire, incarcérée à la Maison d'Arrêt et de Correction de Ouagadougou (MACO))

Une autre enquêtée renchérit «... Si le papa de l'enfant se comporte façon, façon, tu pars avorter... J'ai pris une grossesse et je suis allée dire à mon amant que j'étais enceinte et il m'a frappé... II m'a battu... il y a beaucoup de jeunes qui viennent à la maison, il pense que je couche avec eux... Que ce n'est pas pour lui. "Bordel comme cela. Tu sors avec tes jeunes qui traînent avec toi "... Il m'a frappé, me traîner sur la route, blesser partout... Donc quand je suis partie, j'ai avorté... Que " bordel là, toi tu couches avec les jeunes traînés qui sont ici là... ils t'enceintent et puis tu es là. Je m'en fou. II faut aller accoucher. Ton enfant là, ce n'est pas pour moi hein, c'est pour tes vagabonds là ". Je suis partie enlever" (enquêtée de 36 ans, célibataire, restauratrice).

\section{Les relations illégitimes}

Certaines femmes avortent parce qu'elles sont pressées de régulariser leur situation. Une enquêtée explique le cas d'une femme qui vivait en concubinage avec son partenaire, et qui avorte parce que celui-ci voulait régulariser leur situation. Pressée de saisir l'occasion donnée par son partenaire pour se marier, elle s'adonne à l'avortement car la religion musulmane n'autorise pas la célébration d'un mariage lorsque la femme est en grossesse : «... Chez les musulmans, on dit qu'on ne célèbre pas le mariage quand la femme est enceinte. II faut que tu accouches d'abord. Mais elle, elle disait non, si elle doit attendre le temps d'accoucher, son mari peut changer d'avis et ne plus faire le mariage, repousser encore la date. Donc, qu'est-ce qu'elle a fait ? Elle était à trois mois de grossesse. Elle avait déjà trois enfants. Elle tenait coûte que coûte au mariage. Ils (les musulmans) disent qu'ils ne célèbrent pas le mariage quand la femme est enceinte. Donc c'est cela qu'elle avait avancé comme argument pour avorter. Le mariage, elle avait toujours chanté le mariage, le mariage, le mariage. Au moment où l'homme s'est décidé à célébrer le mariage, il se trouvait qu'elle était enceinte. Donc elle a avorté à l'insu du Monsieur. Elle a fait un choix entre le mariage et puis cet enfants (enquêtée de 40 ans, célibataire, confidente d'une femme qui a avorté).

\section{Les raisons d'ordre économique}

Il ressort de notre travail de recherche que des femmes ont avorté pour des raisons d'ordre économique. La même enquêtée suscitée explique le cas d'une fille qui a avorté parce que son amant ne menait pas d'activité rémunératrice de revenu. Selon les dires de notre enquêtée, c'est la mère de la fille en question qui l'a encouragé à avorter parce qu'ellemême dépendait économiquement de son mari et de ce fait, avait peur de la réaction dudit mari qui allait voir le nombre des personnes à sa charge s'augmenter : "Le jeune n'était pas responsable, mais il disait qu'il voulait l'enfant... II voulait la fille, mais la maman de la fille dit non, pas question... Quand on dit partisan du moindre effort là, c'est un partisan du moindre effort. II ne foutait rien. S'il se lève, c'est pour aller jouer babyfoot, c'est pour aller jouer aux dames... II ne faisait rien, absolument rien. C'est la fille qui était de bonne famille. Donc sa maman a dit net, qu'elle ne veut plus de leur relation. La maman s'est imposée, que c'est vous(les parents de la fille) qui allez prendre en charge la fille, tu as déjà la charge de la fille, tu vas avoir la charge du petit fils encore? Que non, qu'elle ne peut pas... Lui (l'amant), il voyait aussi, il ne pouvait rien faire, il ne pouvait pas s'occuper de l'enfant, il ne pouvait absolument rien faire. Même si on lui disait de payer tant de francs pour l'accouchement, il n'allait pas 
pouvoir payer... La vieille là aussi, elle ne faisait rien. Elle était sous couvert du vieux (dépendait de son mari). Donc il ne faut pas prendre un problème venir ajouter à d'autres problèmes" (enquêtée de 40 ans, célibataire, confidente d'une femme qui a avorté).

La pratique de l'avortement comme moyen de régulation des naissances

Des enquêtées ont avoué utiliser l'avortement comme un moyen de contraception pour espacer leur naissance car elles n'étaient pas en mesure financièrement de s'occuper d'un enfant. C'est le cas de cette femme qui a avorté parce qu'elle était face à une grossesse qu'elle juge trop rapprochée, associée à des difficultés d'ordre économique du couple. II s'agit d'un bébé de deux mois qu'elle avait, et crayait de ne pas pouvoir s'occuper financièrement de deux enfants de bas âge à la fois, vue la situation misérable du couple: "II y a une femme, elle-même elle était mariée. Elle était enceinte mais elle avait un bébé de deux mois. Elle n'arrivait pas à joindre les deux bouts. Donc elle est allée pour avorter... Et vu leur (le couple) situation aussi, pour gagner à manger, c'est difficile, bébé de deux mois est là. Tu vas faire ça là (l'enfant) encore ajouter. Alors qu'entre temps, il faut sevrer l'enfant. Il faut lui donner du lait. Tu n'arrives même pas à bien manger. C'est difficile» (enquêtée de 40 ans, célibataire, confidente d'une femme qui a avorté).

Dans le même ordre d'idées, une enquêtée a fait plusieurs avortements suite à l'échec d'utilisation des méthodes contraceptives. Elle avoue qu'après avoir accouché son premier enfant, elle a choisi d'avorter toute autre grossesse chaque fois que la méthode contraceptive échouait parce qu'elle attendait que son premier enfant grandisse, qu'il soit en mesure de soutenir financièrement son enfant cadet avant d'accoucher : "Quand je suis en grossesse, souvent moi-même je refuse parce que quand tu n'as personne pour te soutenir, tu es seule et puis tu vas faire beaucoup d'enfants, les enfants vont souffrir. Donc moi je préfère avorter... Moi je ne voulais pas faire beaucoup d'enfants parce que je voulais que ma fille grandisse. Si elle a 15 ans ou 16 ans et je fais un deuxième, elle peut..., quand l'autre (nouveau né) va avoir deux ans, elle aura 18 ans comme cela et s'occuper de lui... Moi je prenais les pilules, mais une fois que je me trompe, c'est une grossesse» (enquêtée de 36 ans, célibataire, restauratrice).

La même fille se retrouve actuellement enceinte parce que trouvant son partenaire sexuellement très faible et qui de son avis ne pouvait pas enceinter une femme, n'a pas utilisé de méthode contraceptive. Mais cette fois-ci malgré les difficultés financières qu'elle rencontre et l'insistance de son partenaire à l'inciter à avorter, elle ne tient pas à avorter parce qu'elle désire avoir un deuxième enfant, étant donné que son premier enfant à 15 ans et est censé de ce fait, pouvoir venir en aide à son frère cadet. Au pire des cas, elle préfère accoucher et faire adopter l'enfant par quelqu'un d'autre : "Bientôt ma grossesse aura trois mois, je dois aller à l'hôpital. Je l'appelle, je dis tu ne veux pas me donner l'argent, il faut garder ton argent. II faut envoyer la photocopie de ta pièce là, au moins je vais aller à l'hôpital... "Ton affaire de grossesse là, on a dit d'enlever ». Je dis que je ne connais personne ici et tu veux que j'enlève une grossesse. II faut aller chercher quelqu'un, si tu as trouvé, tu viens me dire et puis je vais partir, mais tu vas assister. Donc quand il parlait, moi-même je vois qu'il parlait trop, trop. Je lui ai dit que je suis allée voir quelqu'un (un avorteur) et il a dit 90000 F. S'il me donnait l'argent, j'allais garder, entretenir la grossesse. II dit je veux faire le commerce. Que " c'est commerce tu veux faire. Je n'en ai pas, il faut accoucher »... Ce n'est pas un problème, s'il ne m'amène pas la photocopie, je vais partir avec la photocopie de la pièce de mon papa, j'ai ça ici... Moi en fait, la grossesse-là, je n'ai pas envie d'enlever sincèrement. Si j'avais envie d'enlever, j'allais enlever, mais je vois que je prends de l'âge et puis j'ai un enfant. C'est à cause de cela que je préfère laisser (garder la grossesse). Je ne vais pas enlever... II me dit d'aller avorter. Je dis non, moi je ne vais pas avorter. Comme je ne vais pas avorter et puis toi tu ne veux pas l'enfant, je vais faire adopter l'enfant. Je préfère aller faire adopter l'enfant » (enquêtée de 36 ans, célibataire, restauratrice).

Le non-respect des clauses définies au début de la relation amoureuse

Une enquêtée justifie son avortement par le nonrespect des clauses définis au début de la relation : " J'étais avec le monsieur et il me disait qu'il me voulait pour mariage. J'ai accepté et j'étais même dans sa cour. C'est lui-même qui a loué la maison pour moi. Quand je suis tombée enceinte, il m'a remis 25 $000 \mathrm{~F}$ dire de partir avorter car il ne veut pas de moi, qu'il m'a flatté, qu'il ne me veut pas pour femme... Que même si j'accouche, quand l'enfant aura 7 ans, il va l'amener dans leur village et je ne le verrai plus jamais. Il m'a dit d'avorter, il m'a forcé remettre 25 $000 \mathrm{~F}$ pour avorter. Moi aussi je suis allée effectivement avorter " (enquêtée incarcérée à la MACO, célibataire, niveau d'instruction $6^{\text {ème }}$.

Les grossesses issues des relations amoureuses occasionnelles sans projet de mariage, ni d'enfant Une minorité de filles a avorté parce qu'elle a entretenu des relations passagères, sans aucun projet de mariage, ni d'enfant avec son amant. C'est le cas 
de cette enquêtée qui ne s'attendait pas à une grossesse et explique la survenue de sa grossesse par une malédiction de la part de son ex-amant, ayant eu un seul rapport sexuel avec un monsieur qu'elle a rencontré pour la première fois : "Mon mari (examant) m'a dit de revenir. Quand il m'a dit de revenir et j'ai refusé, il m'a dit que je vais avoir un problème. Que si je ne trouve pas un problème de la journée, je vais trouver un problème de la nuit. Quand j'ai eu le rapport sexuel avec le monsieur, ce n'était pas suffisant pour que je tombe enceinte... II (ex-amant) a dit que j'allais avoir un problème et effectivement, j'ai eu un problème. II n'y a pas de problème plus grave qu'être dans une prison... Moi je n'ai pas eu plusieurs rapports sexuels avec le monsieur, c'était une seule fois. Mais si quelqu'un t'a maudit, tu peux tomber enceinte... Moi-même je ne sais pas là où le monsieur (partenaire) est... Je ne sais pas s'il continue de vivre ou pas. Aucune personne (les membres de sa famille) ne le connaissait. C'est comme tu es venue aujourd'hui, si tu étais un garçon, on se suit aujourd'hui seulement et c'est fini, tu repars définitivement. Je ne sais plus là où tu es " (enquêtée de 28 ans, célibataire, non scolarisée, incarcérée à la $\mathrm{MACO}$.

\section{Les conséquences de l'avortement provoqué} Les conditions de vie précaires des femmes les exposent aux conséquences néfastes des grossesses non désirées et des avortements provoqués.

\section{Les conséquences sociales de l'avortement provoqué}

Les discours des enquêtés nous ont permis d'avoir une idée claire sur les difficultés que les femmes rencontrent après un avortement provoqué. Concernant les conséquences sociales des avortements provoqués, il est ressorti que les avortements provoqués modifient les relations entre les deux partenaires ou entre la femme et les membres de sa famille, est à l'origine de conséquences fâcheuses qui incitent à la vengeance. Par ailleurs, ces avortements sont sources d'isolement des femmes concernées, de dépenses exorbitantes et peuvent être source de perte d'emploi. Des stigmatisations, violences et sanctions de la part des agents de santé ont été relevées.

La modification des relations entre la femme et les membres de sa famille

La modification des relations entre la femme et les membres de sa famille se perçoit à travers le bannissement des filles de leur famille paternelle, l'isolement des femmes incarcérées suite à l'avortement. Un entretien informel a permis de savoir qu'à cause des interdits coutumiers pour les grossesses hors union, une étudiante a été bannie de sa famille paternelle pour avoir entretenu une relation illégitime qui s'est soldée par une grossesse non désirée et un avortement. En effet, la fille en question aurait tenté un avortement clandestin et suite à des complications, elle se serait rendue dans une des formations sanitaires pour expulser le fœtus, accompagnée par les membres de sa famille qui ignoraient l'origine de sa maladie. C'est dans la formation sanitaire que les agents de santé ont avoué aux membres de sa famille qu'il s'agissait d'un avortement incomplet. C'est ainsi que ceux-ci se sont désengagés. L'étudiante après l'hospitalisation rejoignit sa grand-mère et envoya une de ses amies ramasser ses bagages chez l'oncle paternel chez qui elle logeait pour ses études, ne pouvant plus retourner dans la cour de son oncle, ne serait-ce que pour terminer le reste de l'année scolaire : «... Compte tenu du fait que la famille paternelle a su que j'étais tombée enceinte, de l'hôpital j'ai continué chez ma grand-mère car je ne peux plus aller chez mon oncle " (étudiante en $3^{\text {ème }}$ année d'université, célibataire).

Selon un chef coutumier, le bannissement des filles qui tombent enceinte hors union a pour but de dissuader leurs petites sœurs, afin que celles-ci ne tombent pas dans la même erreur «... Ils allaient à l'extrême pour la bannir. C'est pour les autres (les autres filles qui ne sont pas encore enceintes), ce n'est pas elle-même (celle qui est enceinte). En ce moment, elle va servir d'exemple maintenant pour les autres. C'est un peu méchant et très fort, mais il faut qu'ils partent jusque-là. Là, ils n'avaient pas le choix, puisque s'ils s'amusent, ce qui pourrait se gâter à l'avenir est encore plus énorme, donc ils préfèrent massacrer elle seule (punir sévèrement la fille qui est en grossesse) pour sauvegarder ce qui reste (les autres filles)" (un chef coutumier résidant à Ouagadougou, d'ethnie moaga ayant 68 ans).

La modification des relations entre les deux partenaires sexuels

L'avortement modifie la relation entre la femme et son partenaire sexuel et peut conduire à une séparation. Une enquêtée rapportant les propos d'un monsieur qui justifie sa séparation avec son partenaire sexuelle à cause du fait que celle-ci ait avorté fait savoir: "Le monsieur dit que c'est toimême, que bordel, chienne là, c'est toi-même qui es venue me bousculer, me forcer même à dépenser l'argent pour dire qu'il faut qu'on enlève parce que tu ne peux pas garder la grossesse, parce qu'il faut que je fasse le mariage avant de te prendre... Que justement, c'est parce que tu as fait ça (avorté) même que je t'ai laissé» (Sage-femme de profession d'origine ayant 55 ans). 
Par ailleurs, un entretien réalisé avec un attaché d'éducation spécialisée, exerçant au sein de l'action sociale de la MACO, retrace les démarches qu'il a entreprises auprès du Procureur Général pour qu'il acquitte une femme lors du jugement définitif, ceci pour tenter de sauvegarder le foyer de cette femme qui avait été emprisonnée à la MACO pour raison d'avortement, relâchée pour une liberté provisoire après avoir purgé sa peine et réintégrée plusieurs années en prison pour le jugement définitif. Pour information, la peine de prison va de 6 mois à 2 ans au Burkina Faso. Cette réintégration pour le jugement définitif mettait mal à l'aise son mari et sa belle-famille qui n'avaient jamais su qu'elle avait fait la prison : "J'ai eu un cas, une femme qui avait été incarcérée pour ce problème d'avortement et qui avait été libérée, huit, dix ans plu tard, on devait maintenant l'appeler pour le jugement définitif parce qu'elle avait été en liberté provisoire. Maintenant il s'est trouvé que quand elle est partie, elle s'est mariée. Elle avait même un enfant et la belle famille ne savait pas qu'elle avait été emprisonnée. C'est quand on l'a rappelé pour faire le jugement définitif, on a envoyé la police fouiller la chercher ... Et quand on l'a amené en prison, la belle famille était un peu perdue parce qu'ils (les membres de la famille) ne savent pas pourquoi on vient arrêter leur belle fille. Le mari aussi perdu. Maintenant ils ont su qu'elle avait fait la prison et que le problème était en train de ressurgir pour un jugement définitif. Moi quand j'ai été saisie, j'ai rencontré la belle-famille ... Je me suis beaucoup entretenue avec le mari pour l'amener à comprendre ... Il a fallu que je fasse des démarches à la cour d'appel à Ouaga 2000 auprès du Procureur Général pour soulever ce problème-là, pour dire que sincèrement, pour sauvegarder le foyer de cette femme, il fallait que quelque chose soit fait. Sinon si encore on devrait prononcer les trucs, en tout cas ... A la fin, on a libéré la femme, on a dit le problème était résolu, clos, et la femme a retrouvé son foyer et je disais au monsieur s'il tient vraiment à sa femme et que déjà avec ce qui s'est passé, les voisins ont appris que la femme a fait la prison, ils peuvent déménager, trouver un coin où ils peuvent aller rester tranquille pour que les gens oublient et qu'ils puissent mener leur vie de foyer sans difficulté».

\section{L'Isolement des femmes incarcérées à la MACO pour cause d'avortement}

Les enquêtées qui se sentent isolées selon les résultats de la présente enquête sont celles qui ont été incarcérées à la Maison d'Arrêt et de Correction de Ouagadougou (MACO). Ces interviewées estiment qu'elles sont abandonnées, délaissées par les membres de leur famille et l'entourage : " Je souffre comme cela pour survivre. Je suis ici il y a 10 mois, mais je n'ai jamais mangé un plat de mon choix. Ce sont les gens de la prison qui préparent et qui enlèvent me donner. Personne ne vient me rendre visite. Quand je suis ici là, c'est moi et Dieu... J'ai appelé ma sœur et elle m'a dit qu'elle a appris qu'on incarcère les visiteurs à la MACO. Elle ne veut pas venir parce qu'elle a peur d'être incarcérée. Donc j'ai décidé de ne plus l'appelens (enquêtée de 28 ans, incarcérée à la MACO, non scolarisée).

Une autre enquêtée regrette son emprisonnement à la MACO car elle est isolée de sa famille. Elle a de ce fait, la nostalgie de voir sa famille, surtout sa mère qu'elle n'a plus revue depuis plus de deux ans selon ses dires : "C'est mon grand-frère qui vient ici souvent, mais il y a longtemps de cela il est venu... Ce qui me rend plus triste, c'est le fait que je ne peux pas voir ma maman là seulement. Cela dépasse deux ans. J'avais un oncle paternel aussi qui venait, mais lui aussi il est venu cela vaut presqu'une année maintenant. Je n'ai même plus de ses nouvelles. Quand j'appelle sur son numéro, ça ne passe pas.» (enquêtée non scolarisée ayant 20 ans, incarcérée à la MACO).

\section{Les conséquences fâcheuses incitant à la vengeance}

Des conflits sournois naissent parfois entre la femme qui a avorté et son avorteur suite à l'avortement.

Une sage-femme de profession, ayant 55 ans, bergère de groupe de prière raconte le cas d'une femme qui est venue se confier à elle suite à son avortement et qui était énervée contre son avorteur : « Elle dit qu'en tout cas, que l'infirmier-là, je dis mais, tu le reconnais toujours? Est-ce que vous vous voyez ? Ou bien c'est une personne âgée qui n'existe plus ? Que non, que l'imbécile est toujours là. Qu'il est toujours là. Que des fois même quand elle, elle passe vers le quartier-là, vers la route-là, quand elle voit la maisonnette-là, que ça lui fait mal, elle a envie de s'arrêter pour l'insulter ».

Cette idée se confirme par le discours de l'avorteur clandestin que nous avons interviewé. En effet, l'avorteur clandestin se plaint du fait que les femmes qu'il a avortées ne sont pas reconnaissantes à son égard : "C'est un travail ingrat car après l'acte d'avortement, les femmes ne sont pas reconnaissantes... Quand j'avais ma clinique, si tu vois le nombre de femmes que j'ai aidé là (l'aide consiste au fait qu'il les a avorté), mais elles ne sont pas reconnaissantes. La médecine même est ingrate. Les femmes que j'ai aidé ne passent pas après me rendre visite, elles ne m'appellent pas non plus. Si une femme m'appelait me dire: "Docteur, j'ai eu la santé ", je serai contents (propos d'un avorteur clandestin ayant 60 ans, niveau d'instruction BEPC). 
L'avortement comme une source de perte d'emploi

L'avortement peut aussi être une source de perte d'emploi. Une fille qui a avorté clandestinement, dénoncée à la police, a été incarcérée à la MACO pour 6 mois de prison ferme et menace de perdre son boulot : "Le Directeur des Ressources Humaines a dit que je perdrai mon emploi si toutefois je fais trois mois sans venir... Par rapport à mon travail, je doute car c'est un (nationalité de l'employeur) et il licencie les gens au hasard. Les (nationalité de l'employeur) ne tardent pas à licencier les gens) (une fille ayant 29 ans incarcérée à la MACO, célibataire de niveau d'instruction $3^{\text {ème }}$, Gérante dans une boulangerie de la place).

\section{L'avortement comme une source de dépenses exorbitantes}

Des enquêtées ont présenté l'avortement comme une source de dépenses exorbitantes. Nous en voulons pour preuve une interviewée qui trouve anormal que pour son arrestation, les autorités intervenant dans ce domaine lui fassent faire les dépenses : " La gendarmerie m'a conduit à Pôle 6 (formation sanitaire), mais ils (les gendarmes) n'ont pas payé les frais. J'ai payé $7000 \mathrm{~F}$ pour l'échographie au collège protestant. Ils disent de voir un médecin, là aussi j’ai payé $5000 \mathrm{~F}$. Les dépenses s'élevaient à plus de $20000 \mathrm{~F}$ en dehors des $100 \mathrm{~F}$, $100 \mathrm{~F}$, payé pour le parking. Comme eux, ils veulent faire des enquêtes, ce sont eux qui devraient faire les dépenses et m'arrêter. Ce n'est pas normal ... C'est moi qui ai fait toutes les dépenses ... Dans les normes, c'est la gendarmerie qui devrait faire ça. J'ai dépensé plus de $40000 \mathrm{~F}$. Ils m’ont amené à la maternité et comme la maternité ne leur a pas donné de conclusion sur ce qui est arrivé, ils ont demandé une échographie et dire de voir le médecin, alors qu'ils n'ont pas pris en charge les dépenses " (se plaint une fille de 29 ans incarcérée à la MACO, niveau d'instruction $3^{\text {ème }}$ ).

Des stigmatisations, violences et sanctions de la part des professionnels de la santé

Des stigmatisations, violences et sanctions de la part des agents de santé ont été relevées. Nous en voulons pour preuve les propos de cette sage-femme parlant d'une sanction affligée à une jeune fille qui a tentée au préalable un avortement clandestin, et qui s'est retrouvée dans un centre de santé confessionnel pour expulser le fœtus suite à une complication: "Quand j'étais à (nom de la formation sanitaire) par exemple, il y a une fille qui a avorté une grossesse de six mois. Et quand elle a avorté, c'était à ma garde... Et comme c'est dirigé par les sœurs, une sœur religieuse, quand elle est arrivée, on a expliqué le cas.
Elle a pris la fille et le fœtus aller enfermer dans son bureau. Elle a pris un truc tout blanc, c'est des couches toutes blanches qu'on avait, elle a mis le fœetus dedans, la fille s'est assise, elle a déposé le fœetus sur ses pieds. Moi je suis descendue en tout cas les laisser là-bas. II paraît que la fille est restée pendant près de cinq heures de temps enfermée dans le bureau de la Sœur avec le fœtus. Elle a pleuré, elle a pleuré. Elle a regretté son acte... C'était en fait pour la choquer quoi, pour que de sa vie-là, elle ne recommence plus jamais» (propos rapporté par une sage-femme de profession ayant 50 ans, parlant d'une patiente qu'elle a reçue lorsqu'elle travaillait dans une maternité confessionnelle de la place).

\section{Les conséquences psychologiques de l'avortement provoqué}

Au cours de nos investigations, plusieurs symptômes dépressifs ont été rapportés par les femmes qui ont avorté (regret, honte, douleurs morales, insomnies, crainte, incertitude par rapport à une future grossesse, sentiment de désespoir).

\section{Le sentiment de regret}

Le regret a été évoqué par un nombre important de femmes qui a avorté clandestinement. C'est le cas de cette enquêtée qui a des insomnies suite à son avortement : "Ce qui m'empêche de dormir est que je pense beaucoup maintenant et je me dis que si je savais, je n'allais pas avorter ... Même si on ne me condamnait pas, je ne pouvais pas ne pas regretter l'acte que j'ai posé, parce que c'est une vie que j'ai ôtée. Moi-même je sais que ce n'est pas bien " (enquêtée de 20 ans, non scolarisée, incarcérée à la MACO).

Une autre renchérit : "Maintenant je réfléchis, quand ma maman était enceinte de moi, si elle avait avorté, je ne serais pas en vie présentement. Ce dont je pense et ça me fait souffrir, c'est cela. Ce dont je réfléchis et ça me traumatise, c'est cela. Si ma maman m'avait traité de cette façon, je ne serais pas en vie actuellement " (enquêtée de 28 ans incarcérée à la MACO, non scolarisée).

Pour certaines femmes qui ont avorté, le regret vient du fait qu'en vue de bénéficier de l'aide de l'avorteur clandestin, elles ont été victimes d'abus sexuel. En effet, après avoir introduit les comprimés dans l'utérus de la femme qui veut avorter, cet avorteur propose de coucher avec elle avant la procédure pour que les comprimés aillent à l'intérieur de l'utérus selon lui. Une enquêtée faisant savoir les propos d'une de ces femmes qui a eu du remords après qu'elle ait été avortée clandestinement par l'avorteur raconte : "Celui qui était à la (quartier où habite l'avorteur) qui faisait l'avortement jusqu'à ce qu'on l'arrête là, lui quand il 
introduit le produit, il couche avec les filles ... C'est un imbécile, sinon s'il introduit le produit, il n'avait pas besoin de les coucher. Le produit pouvait monter seul ... Une femme était enceinte et elle avait un bébé de deux mois ... Elle n'arrivait pas à joindre les deux bouts. Donc elle est allée pour avorter et le gars lui dit je couche avec toi pour que le produit-là aille à l'intérieur. Comme elle était dans l'obligation, elle a accepté. Mais elle, elle a regretté. Elle a regretté. Quand elle voyait son mari, elle avait honte ... Elle a vu qu'elle avait fait l'adultère puisque son mari n'était pas au courant de cela et elle ne peut pas lui dire ... Cette femme, elle a été choquée. C'est elle qui a avorté et encore elle a commit l'adultère... Et puis comme elles sont en position de faiblesse, elles ne refusent pas, le problème est là̀ (enquêtée ayant 40 ans, coiffeuse, célibataire de niveau $d^{\prime}$ 'instruction $5^{\text {ème }}$ (des Lycées et collège).

Beaucoup d'interviewées qui ont avorté voyaient en l'avortement, une offense faite à Dieu et se rependaient : " J'ai beaucoup avorté quand j'étais en Côte d'Ivoire ... En tout cas, j'ai l'habitude de faire (avorter) ... Moi je ne change pas de Docteur ... Quand je dis Docteur, il dit : "Tu veux faire (avorter)?". Je dis oui. "Viens"... En tout cas, j'ai trop fait (avorté) sincèrement. Je demande pardon à Dieu» (enquêtée de 36 ans, célibataire, restauratrice). Selon les propos de sa confidente qui m'a mise en contact avec qu'elle, elle aurait avorté 7 fois.

\section{La douleur morale}

La douleur morale se manifeste par la tristesse, les larmes aux yeux. Une enquêtée profondément blessée a fondu en larmes tout au long de l'interview qui a duré une quinzaine de minutes parce qu'elle a été incarcérée pour avoir avorté : "Depuis que je suis ici, je n'ai jamais dormi la nuit (pleurs). Je suis allée leur (les gendarmes) raconter mon problème et ils m'ont condamnée (pleurs) ... Ils (les gardes de sécurité pénitentiaire) disent qu'ils vont me condamner durant deux ans. Ce n'est pas à l'enfant (fœtus avorté) que je pense, c'est parce qu'on m'a incarcérée que je n'arrive pas à dormir " (prisonnière à la MACO, célibataire ayant 31 ans, niveau d'instruction $6^{\text {ème }}$ ).

\section{Le sentiment de honte}

Des femmes interviewées sous l'effet de la honte et pour se soulager moralement, fuient leur responsabilité. Une interviewée déclare à cet effet que le fœtus qu'elle a avorté n'était qu'un amas de cellules : "Si tu veux faire un avortement, c'est mieux de faire très tôt. Je pense que c'est le sang seulement. Sinon si toi tu laisses ça devient comme un enfant dans ton ventre et puis tu te lèves pour aller faire cela (avorter), c'est comme si tu as tué un homme. Voilà, ça aussi ce n'est pas bon. Par exemple moi, si je sais que je ne veux pas une grossesse, je ne vais pas laisser la grossesse arriver quatre mois. Tu sais, quatre mois-là, c'est déjà un homme. Mais si c'est un mois, deux mois, deux mois et demi, c'est comme c'est toujours le sang. Là, tu peux faire couler ça (avorter). Je sais que ce n'est pas bien, mais moi je me dis que le temps que c'est toujours le sang, tu peux faire couler et Dieu peut te pardonner en ce moment. Mais si ça s'est formé jusqu'à ce que ça devienne comme un enfant, vraiment, ça peut jouer sur ta vie parce que tu te dis que tu as tué un être humain. Pour faire l'avortement, c'est mieux de faire très tôt, pendant que la grossesse n'a pas encore pris la forme d'un être humain. Laisser cinq mois, quatre mois, six mois aller avorter là, ce n'est pas bon ... Moi, j'ai avorté pour moi à deux mois. Ça, c'est le sangs se défend une enquêtée de 32 ans, de niveau d'instruction $5^{\text {ème }}$.

\section{L'Incertitude par rapport à une future grossesse}

L'avortement a été présenté comme une source d'incertitude par rapport à une future grossesse. A ce sujet, une étudiante de 27 ans ayant bénéficié de soins post avortement craint de ne pas pouvoir enfanter parce qu'elle a perdu une de ses trompes et doit subir une deuxième intervention en vue de déboucher la trompe restante. A la question de savoir si sa santé s'est améliorée, elle laisse entendre : " ça va où ça ? Est-ce que tu sais que je dois faire une deuxième intervention pour déboucher la deuxième trompe? Lors de la première intervention, l'autre trompe avait été enlevée ... La fille qui est dans notre cour là, elle a fait un avortement. J'avais envie de lui dire de ne pas faire, mais elle ne parle pas avec moi. Elle ne sait pas. Les nous autres là, notre cas est bizarre, ce n'est même pas sûre qu'on va enfanter ".

\section{Le sentiment de désespoir}

II faut noter qu'un grand nombre de femmes qui a avorté est désespéré car elle croit que l'avortement est le commencement d'une déchéance psychologique. C'est ce qu'avoue cette fille incarcérée à la MACO pour raison d'avortement clandestin : "Si tu es ici (MACO), tu ne peux pas ne pas penser. Pourquoi vas-tu penser? Tu vas penser parce que ta vie est gâchée. A ta sortie de la prison, toutes les personnes qui devraient collaborer avec toi vont te fuir. Ils vont dire que telle personne a fait la prison. Cela gâte le nom. Mais si Dieu dit tu vas faire ceci (avorter et faire la prison), tu ne peux pas échapper à ton destin " (une prisonnière de 28 ans, célibataire, non scolarisée). 
Force est de savoir qu'à côté de ces sentiments d'insatisfaction, une minorité d'enquêtés trouve en l'avortement provoqué un salut.

\section{Le sentiment de satisfaction}

Contrairement à la quasi-totalité des femmes interviewées qui regrettent d'une manière ou d'une autre leur avortement, une femme qui a fait un avortement thérapeutique trouve en l'avortement provoqué un salut. C'est ce que pense cet enquêté qui avorte les femmes à l'aide de produits traditionnels, qui a conduit une fille chez un agent de santé pour son avortement sous prétexte que l'âge de la grossesse était avancé et par conséquent, dépassait ses compétences. La fille en question aurait commis cet acte dans le but de sauver sa relation amoureuse avec un de ses partenaires qui lui avait promis le mariage. L'avortement a permis à cette fille de se marier avec ce partenaire : «... Si le monsieur avait su, est-ce qu'il allait la marier? Même si c'est toi ! Si le monsieur avait su, il n'allait pas la marier. Mais comme elle a avorté discrètement, elle est mariée. Elle a deux enfants " (un charlatan non scolarisé).

En outre, au-delà des femmes interwiewées qui ont avorté clandestinement, une femme qui a fait un avortement thérapeutique a été interrogée. Cette interviewée ressentait de la gratitude à l'endroit de son médecin avorteur : "Compte tenu du fait que ma santé était menacée, les agents de santé ont préféré sacrifier la vie de l'enfant pour sauver celle de sa mère car dans ces conditions, c'est la vie de la mère qui est prioritaire. J'ai eu la chance ... Depuis ce temps, quand je suis enceinte, c'est lui (le médecin qui a fait l'avortement) qui m'assiste» (enquêtée de 40 ans, restauratrice, ayant un niveau d'instruction CE2).

\section{DISCUSSION}

\section{Limite de l'étude}

Le présent travail de recherche a abordé un sujet sensible, fortement stigmatisé dans le contexte Burkinabè. Ce qui n'a pas été sans avoir une influence sur la collecte des données. D'une part, certaines femmes qui ont avorté ont refusé de participer à l'enquête. II a fallu s'armer de patience, agir avec tact pour pouvoir cibler le maximum de femmes ayant avorté et pouvant nous fournir des informations sur les centres d'intérêts explorés. D'autre part, nous avions souhaité rencontrer toutes les femmes ayant avorté citées par leurs confidentes sans intermédiaire, afin de comprendre au mieux les causes et les conséquences de leur avortement. Mais certaines confidentes n'ont pas pu nous mettre en contact avec ces femmes de peur d'affecter négativement leur relation avec ces dernières. Nous nous sommes contentés donc des propos rapportés par leurs confidentes, sachant que comme on le dit souvent dans un langage populaire, "traduire c'est trahir».

Par ailleurs, nous avons eu des difficultés à obtenir les aveux de quelques femmes qui ont avorté. C'est le cas d'une fille incarcérée à la MACO pour une interruption volontaire de grossesse et qui a nié avec la dernière énergie les faits qui lui ont été reprochés lors de l'entretien.

\section{Déterminants de l'avortement}

Avortement comme moyen de régulation de la fécondité

Des femmes interviewées dans la présente étude ont avoué utiliser l'avortement comme un moyen de contraception pour réguler leur naissance, soit à cause du fait que la grossesse est trop rapprochée ou que l'enquêtée juge qu'elle n'a pas les moyens en ce moment précis pour s'occuper d'un enfant qui doit naître. Ces résultats sont similaires à ceux de Desgrées Du Lou et al. (1999 : 440) qui ont présenté l'avortement comme une méthode de régulation de la fécondité au cours d'une enquête menée à Abidjan en 1997 auprès de $120 \mathrm{I}$ femmes enceintes venues en consultation prénatale dans des formations sanitaires urbaines. L'étude menée par Guillaume (2003 : 756), ne déroge pas à cette règle. Les résultats revelaient que certaines femmes avaient expliqué leur avortement par un besoin de limiter (3 $\%)$ ou d'espacer ( $9 \%$ ) les naissances, exprimé par le jugement que "le dernier enfant était trop petit». Pour ces femmes, avortement et contraception sont deux outils de régulation de la fécondité utilisés de façon complémentaire. Son enquête avait révélé que (30 \%) des femmes avaient utilisé à la fois la contraception et l'avortement pour éviter toute grossesse non prévue ou non désirée.

Toujours en Côte d'lvoire, d'autres auteurs comme Benie Bi Vroh et al. (20I2 : 67 et 70) ont également trouvé une liaison entre l'utilisation des contraceptifs et le recours à l'avortement. Étant donné que l'utilisation d'une contraception traduit le désir des femmes de ne pas débuter de grossesse, leur attitude serait de recourir fréquemment à l'avortement en cas de grossesses non désirées ou d'utiliser la contraception suite à un avortement. Dans leur travail de recherche, le taux des femmes qui avaient avorté par manque de ressources financières pour assurer la charge d'un enfant était de $(21,3 \%)$. Or, l'avortement tel qu'il est décrit au paragraphe 8.25 du rapport de la Conférence internationale sur la population et le développement, " ne devrait, en aucun cas, être promu en tant que méthode de planification familiale (Nations Unies, 1994 : 57). 
Conséquences sociales des avortements

La modification des relations entre les deux partenaires sexuels

Des résultats de la présente étude ont montré que l'avortement modifie la relation entre les deux partenaires sexuels et peut conduire à la séparation. Bien avant cette étude, une enquête qui avait été menée dans la région Ostschweiz en suisse avait souligné cet état des faits. En effet, Stössel (1992 : 85 ) avait soutenu dans leur étude que $70 \%$ des couples interrogés s'étaient séparés dans l'année qui avait suivi l'avortement, qu'ils aient été mariés ou non.

La présente étude a également fait savoir que l'avortement modifie les relations entre la femme et les membres de sa famille. Ces modifications se perçoivent à travers le bannissement des filles fautives de leur famille paternelle et l'isolement pour les femmes incarcérées suite à l'avortement. Ces résultats rejoignent ceux de N'Bouke (201 I : 136) qui traitant du recours à l'avortement provoqué à Lomé au Togo expliquait que sur le plan relationnel, $73,7 \%$ des femmes avaient déclaré que leur dernier avortement avait entrainé des problèmes entre elles et leurs parents, spécialement entre la femme et son père.

\section{L'avortement comme une source de dépenses exorbitantes}

Des résultats de notre étude ont relevé que l'avortement est une source de perte de revenus, une source de dépenses exorbitantes. Ces résultats sont similaires en quelque sorte à ceux de llboudo (2014 : 86) qui soutenait que les paiements associés aux avortements provoqués et spontanés étaient élevés et pouvaient causer un appauvrissement à court terme ou à long terme des femmes qui bénéficiaient des soins, en particulier pour les femmes ayant fait des avortements provoqués. En effet, le traitement des complications d'avortement provoqué semblait consommer une part importante des ressources du ménage. La seule différence des dépenses effectuées par les femmes qui ont avorté dans les deux travaux de recherches, est que dans le cadre de l'enquête de Ilboudo, les femmes dépensaient pour se soigner. Dans la présente étude, les dépenses associées aux avortements sont effectuées pour se rassurer que les femmes ont effectivement avorté et si oui, les emprisonner.

Des stigmatisations, violences et sanctions suite aux avortements

Des résultats de la présente recherche ont montré que des femmes qui ont avorté subissent des stigmatisations, violences et sanctions suite à leur avortement. Nos résultats contrastent avec ceux d'une étude conduite à Amsterdam au Pays-Bas. Les résultats de cette étude avaient fait savoir que l'avortement n'est pas perçu comme une source de stigmatisation dans la mesure où le stigmate de l'avortement n'est pas visible. En effet, tant qu'une femme qui a avorté ne le dit pas, l'on ne peut pas à vue d'œil savoir qu'elle a avorté. Ce qui amoindrirait le stigmate de l'avortement comparativement à celui d'autres maux visibles qui minent la société. La stigmatisation de l'avortement n'est ni naturelle, ni « essentielle " (Kumar et al., 2009: 629, 630 et 639).

\section{Conséquences psychologiques des avortements}

Au cours de cette enquête, plusieurs symptômes dépressifs liés à l'avortement provoqué ont été rapportés par les femmes qui ont avorté elles-mêmes et par leurs confidentes. Ce sont les douleurs morales, les insomnies, la crainte, l'incertitude par rapport à une future grossesse, des sentiments de désespoir, de regret et de honte. Ce qui nous amène à affirmer que l'avortement n'est pas uniquement un problème de santé, mais aussi un problème de société. Ces résultats s'apparentent à ceux trouvés par N'Bouke (20II: 135) qui faisait savoir que compte tenu du contexte de stigmatisation de l'avortement au Togo, plusieurs femmes gardent des séquelles au niveau psychologique selon ce qu'elles ont ressenti après leur avortement. Les résultats montraient que $77,9 \%$ des femmes avaient eu des regrets ou remords après leur dernier avortement et environ 3,3\% avaient ressenti de la culpabilité. En outre, Ky (1998 : 58), qui avait mis l'accent sur les aspects épidémiologiques et cliniques de la prise en charge des avortements dans les maternités de la ville de Ouagadougou avait fait remarquer bien avant que le coût des avortements renvoie aussi aux coûts non estimables tels que le coût social et les séquelles psychologiques.

\section{Sentiment de satisfaction}

Contrairement à la quasi-totalité des femmes interviewées lors de cette enquête qui regrettent d'une manière ou d'une autre leur avortement, une minorité de ces femmes ont trouvé en l'avortement un salut. Des résultats semblables avaient été mentionnés dans la thèse de N'Bouke (201 I : 136). En effet, des sentiments moins négatifs étaient aussi parfois ressentis après avortement. Ainsi, $10,5 \%$ des femmes ayant avorté au moins une fois avaient ressenti une certaine indifférence et $3,8 \%$ avait eu un soulagement après leur plus récent avortement.

\section{Conclusion}

Au terme de cette étude, il ressort que les causes des avortements sont notamment les relations illégitimes, 
les raisons d'ordre économiques, les grossesses trop rapprochées, le refus de paternité, le non-respect des clauses définies au début de la relation et les grossesses issues de relations occasionnelles sans aucun projet de mariage, ni d'enfant. Quant aux conséquences, elles se subdivisent en conséquences sociales qui se résument comme suit : modification des relations entre les deux partenaires sexuels ou entre la femme et les membres de sa famille, provocation de conséquences fâcheuses incitant à la vengeance, isolement des femmes ayant avorté, sources de dépenses exorbitantes, de perte d'emploi, de stigmatisations, violences et sanctions de la part des agents de santé. Les conséquences psychologiques sont : plusieurs symptômes dépressifs (regret, honte, douleurs morales, insomnies, crainte, incertitude par rapport à une future grossesse, sentiment de désespoir). Des sentiments de satisfaction ont été aussi rapportés par une minorité d'enquêtés.

Notons que si les causes des avortements mises en exergue dans cet article sont quasiment similaires à celles d'autres études menées à Ouagadougou, les données portant sur les conséquences sociopsychologiques avaient été rarement documentées. En effet, des données particulières comme celles portant sur les femmes incarcérées, les avorteurs, les conflits sournois entre avorteurs et avortés font l'originalité de cet article.

Les effets sociaux et psychologiques néfastes des avortements sont considérables et nécessitent une prise en charge des femmes en tenant compte de leurs besoins. II est de ce fait nécessaire de prévoir une prise en charge incluant les aspects médicaux, sociaux et psychologiques.

Le ministère de la santé est invité à créer une structure de prise en charge psycho-sociale, prévoir des services de santé mentale afin de prendre en charge les femmes qui avortent. Cette structure permettra de donner des conseils appropriés aux femmes. Ce qui va leur permettre d'éviter les grossesses non désirées et par ricochet, réduire la nécessité de recourir à l'avortement. Cette structure par sa prise en charge psycho-sociale permettra également aux femmes qui ont avorté de ne pas tomber dans la dépression.

En outre, ce ministère doit œuvrer à renforcer la sensibilisation sur l'utilisation des méthodes contraceptives en vue d'améliorer les services de planification familiale. Les agents de santé qui interviennent dans le domaine de la santé de la reproduction doivent être formés davantage en vue d'améliorer leur attitude à l'égard des femmes qui avortent.

Il est nécessaire par ailleurs de prendre des mesures pour atténuer les sanctions contre les femmes qui avortent de façon clandestine, notamment en ce qui concerne la réinsertion de ces femmes à la MACO pour un jugement définitif plusieurs années après leur avoir accordé une liberté provisoire.

\section{REFERENCES BIBLIOGRAPHIQUES}

African Population and Health Research Center, Ministry of Health (Kenya), Ipas. \& Guttmacher Institute. (20/3). Incidence and complications of Unsafe Abortion in Kenya: key Findings of a National Study. Nairobi, Kenya, 32 p.

Assemblée Nationale. Loi n049-2005/AN 2I Décembre 2005 portant Santé de la reproduction. Burkina Faso, Décembre 2005

Bakass, Fatima, et Ferrand, Michèle (2013). L'entrée en sexualité à Rabat : les nouveaux « arrangements » entre les sexes. Population (Vol. 68-n I), 4I-65.

Bankole, Akinrinola, et al. (20|3). Grossesse non désirée et avortement provoqué au Burkina Faso: causes et conséquences. New York: Guttmacher Institute, rapport, $40 \mathrm{p}$.

Benie Bi Vroh, Joseph, et al. (20I2). Épidémiologie des avortements provoqués en Côte d'lvoire. Santé Publique, HS (Hors Série) (24), 67-76.

Centre pour la gouvernance démocratique. (2010). Les Burkinabés et leurs valeurs : enquête CGD sur les valeurs. Ouagadougou, Burkina Faso, rapport, $66 \mathrm{p}$.

Dao, Alimatou. Lala. (20I0). Soins après avortement: Bilan de II années de pratique dans le service de gynécologie obstétrique du CHU-YO du ler Octobre 1997 au 31 Décembre 2007. Ouagadougou, Université de Ouagadougou, thèse de doctorat en médecine, 88 p. + annexes.

Desgrées du Loû, Annabel. (1999). Le recours à l'avortement provoqué à Abidjan : une cause de la baisse de la fécondité?. Population (54e année, $\left.n^{\circ} 3\right), 427-446$.

Direction des Etudes et de la Planification (DEP). (20I2). Analyse synthétique des résultats de l'énumération de la population de Ouagadougou de 20I2. Ouagadougou, Burkina Faso, rapport, 20 p.

Direction générale des études et des statistiques sectorielles du ministère de la santé. (2015). Annuaire statistique 20I4. Ouagadougou, Burkina Faso, rapport, $317 \mathrm{p}$.

Guiella, Georges. (2004). Santé sexuelle et de la reproduction des jeunes au Burkina Faso : un état des lieux. Occasional Report (No. 12), New York : The Alan Guttmacher Institute, 37 p. + annexes.

Guillaume, Agnès. (2003). Le rôle de l'avortement dans la transition de la fécondité à Abidjan au 
cours des années 1990. Population, (Vol. 58-n6) 74I-772.

Ilboudo, Patrick. Gueswendé. Christian. (20/4). Abortion in Burkina Faso: key determinants, costs, and consequences. Thesis of the degree of Doctor of Philosophy, University of Oslo, Faculty of Medicine, $86 \mathrm{p}$

Institut National des Statistiques et de la Démographie. (2009). Etat et structure de la population. Recensement Général de la Population et de l'Habitat (RGPH) de 2006. Analyse des résultats définitifs. Ouagadougou, Burkina Faso, rapport, $180 \mathrm{p}$.

Kellerhals, Jean. (1977). La négociation sociale de l'avortement ; matériaux pour une construction d'objet. Déviance et société, (Vol. I -n³). 327339.

Kumar, Anuradha, et al. (2009). Conceptualising abortion stigma. Culture, Health \& Sexuality (Vol.II -n 6), 625-639

Ky, André. Yolland. (1998). Les avortements dans les maternités de la ville de Ouagadougou: aspects épidémiologiques et cliniques. Prise en charge. Ouagadougou, Université de Ouagadougou, thèse de doctorat en médecine, $108 \mathrm{p}$.

Ministère de la santé. (20I I). Supporting the use of research evidence (sure) for policy in african health system. Ouagadougou: Note de politique SURE. Ouagadougou, Burkina Faso, rapport final, 7I $\mathrm{p}$.

Nations Unies. (1994). Rapport de la conférence internationale sur la population et le développement : Le Caire. Nations Unies, 194 p.

N'Bouke, Afiwa. (20ll). Recours à l'avortement provoqué à Lomé (Togo) : évolution, facteurs associés et perceptions. Université de Montréal, thèse de doctorat en démographie, $196 \mathrm{p}+$ annexes.

N'Bouke, Afiwa, et al. (20/2). "Le recours à l'avortement provoqué à Lomé (Togo) " Évolution et rôle dans la réduction de la fécondité. Population (Vol. 67-n²), 357-385

Olivier de Sardan, Jean.-Pierre. (2005). La rigueur du qualitatif: Les contraintes empiriques de l'interprétation socio-anthropologique. Louvain-laNeuve : Academia Bruylant, 365 p.

Ouandaogo, Boubakar. (20I5). Soins après avortements au département de Gynécologie obstétrique et médecine de la reproduction du centre hospitalier universitaire Souro Sanou de Bobo-Dioulasso de 2008 à 2013. Ouagadougou, Université de Ouagadougou, thèse de doctorat en médecine, $80 \mathrm{p}$.

Ouédraogo, Ramatou. (2015). "L'avortement, ses pratiques et ses soins " une anthropologie des jeunes au prisme des normes sociales et des politiques publiques de santé au Burkina Faso. Thèse de doctorat en anthropologie socialeethnologie, Université de bordeaux, $466 \mathrm{p}$.

Ouédraogo, Wendwaoga. Salim. Madina. (20I4). Les avortements provoqués clandestins avoués: Profil des patientes, aspects cliniques, thérapeutiques et pronostiques. A propos de I46 cas au service de gynécologie et d'obstétrique du centre hospitalier universitaire Yalgado Ouédraogo. Ouagadougou, Université de Ouagadougou, thèse de doctorat en médecine, $69 \mathrm{p}$.

Organisation Mondiale de la Santé (OMS). (2004). Avortement médicalisé : Directives techniques et stratégiques à l'intention des systèmes de santé. Genève, 106 p.

Palenfo, Babalmana. (20I5). Insuffisance rénale aiguë au cours de l'avortement provoqué clandestin au Centre Hospitalier Universitaire Yalgado Ouedraogo (CHU-YO) de 2010 à 2014. Ouagadougou, Université de Ouagadougou, thèse de doctorat en médecine, $87 \mathrm{p}$.

Quivy, Raymond, et Van Campenhoudt Luc. (1995). Manuel de recherche en sciences Sociales. Paris: Dunod, 287 p.

Rossier, Clémentine, et al. (2006). Estimating clandestine abortion with the confidants' method. Results from Ouagadougou, (Burkina Faso). Social Science and Medicine (62), 254-266

Sedgh, Gilda, et al. (20l I). Estimating Abortion Incidence in Burkina Faso Using Two Methodologies. Studies in Family Planning (Vol. 42 $\left.-n^{\circ} 3\right), 147-154$

Société biblique de Genève. (2007). La Bible. Génève, Segond 21, L'original, avec les mots d'aujourd'hui, $822 \mathrm{p}$.

Stössel, Pius. (1992). Myriam..., pourquoi pleurestu? Les souffrances des femmes après un avortement, Etudes et rapports de médecins sur les séquelles psychiques de l'avortement. Ouvrage original en allemand traduit en français, I54 p.

Winkin, Yves. (1999). Anthropologie de la communication: De la théorie au terrain. Paris: Bruxelles, $239 \mathrm{p}$.

Zongo, Sylvie, et al. (20/5). Les déterminants socioculturels et communautaires des grossesses non désirées et des avortements. Ouagadougou, rapport d'étude, $69 \mathrm{p}$. 